\title{
Estágio extracurricular no setor de arquivo médico e estatística e no núcleo interno de regulação de um hospital público: relato de experiência
}

\author{
Extracurricular internship in the medical and statistical archive sector and in the internal \\ regulation center of a public hospital: experience report
}
Pasantía extracurricular en el sector de archivo médico y estadístico y en el centro de regulación interna de un hospital público: informe de experiencia

José Benedito dos Santos Batista Neto ${ }^{1 *}$, Livia Caroline Machado da Silva1, Caio Heitor Vieira Melo, Ana Gabriela Sabaa Srur de Andrade², Xaene Maria Fernandes Duarte Mendonça ${ }^{2}$, Pilar Maria de Oliveira Moraes ${ }^{2}$, Ailson Almeida Veloso Júnior ${ }^{1}$, Benedito do Carmo Gomes Cantão ${ }^{1}$, Jaylen França Cunha1, Thiago Marcírio Gonçalves de Castro'.

\section{RESUMO}

Objetivo: Relatar a experiência vivenciada por acadêmicos do curso de Enfermagem em um Estágio Extracurricular no Setor de Arquivo Médico e Estatística (SAME) e no Núcleo Interno de Regulação (NIR) de um hospital do interior do estado do Pará. Relato de experiência: Trata-se de um estudo descritivo, do tipo relato de experiência, sobre a atuação de discentes do curso de Enfermagem em um Estágio Extracurricular que foi desenvolvido em dois setores, SAME e NIR, de um Hospital Público. No que diz respeito às ações desenvolvidas pelos estagiários, foi primeiramente elaborado dois manuais para os setores, o "Manual de Normas e Rotinas e Regimento Interno do NIR" e o "Manual de Normas e Rotinas e Regimento Interno do SAME". Ademais, dentro do SAME, os estagiários realizaram atividades nos Setores de: Arquivo, Registro e Internação e Estatística, já dentro do departamento do NIR, as ações foram baseadas na regulação do acesso aos serviços hospitalares, incluindo gestão de leitos e transferências externas através dos sistemas de informação e de regulação dos leitos municipais e estaduais. Considerações finais: Concluiu-se que o estágio extracurricular proporcionou aos acadêmicos uma experiência ímpar, fazendo-os adquirir conhecimentos e habilidades que serão de grande valia em suas vidas profissionais futuras.

Palavras-Chave: Arquivos, Regulação, Enfermagem, Organização e administração.

\section{ABSTRACT}

Objective: To report the experience of undergraduate Nursing students in an Extracurricular Internship in the Medical and Statistical Archive Sector (MSAS) and Internal Regulation Center (IRC) of a hospital in the interior of Pará. Experience report: It is a descriptive study, of the experience report type, about the performance of Nursing students in an Extracurricular Internship that was developed in two departments, MSAS and IRC, of a Public Hospital. Regarding to the actions developed by the interns, first it was prepared two manuals for each sectors "Rules and Routines Manual and Internal Regiment" for both MSAS and IRC. In addition, in MSAS, interns performed activities in the Archive, Registration and Hospitalization and Statistics, in MSAS, interns performed activities in the Archive, Registration and Hospitalization and Statistics Sector, and in IRC, the actions were based on the regulation of access to hospital services, including management of hospital beds and external transfers through information and regulation systems of municipal and state hospital beds. Final considerations: It was concluded that the extracurricular internship provided the students with a unique experience, making them acquire knowledge and skills that will be of great value in their future professional lives.

Keywords: Archives, Regulation, Nursing, Organization and administration.

\footnotetext{
${ }^{1}$ Universidade do Estado do Pará (UEPA), Tucuruí - PA. *E-mail: netto1443@gmail.com

2 Fundação Santa Casa de Misericórdia do Pará (FSCMP), Belém - PA.
} 


\section{RESUMEN}

Objetivo: Informar sobre la experiencia de estudiantes de pregrado de enfermería en una pasantía extracurricular en el Sector de Archivo Médico y Estadístico (SAME) y el Centro de Regulación Interna (CRI) de un hospital en el interior del estado de Pará. Informe de experiencia: Es un estudio descriptivo, del tipo de informe de experiencia, sobre el desempeño de los estudiantes del curso de Enfermería en una pasantía extracurricular que se desarrolló en dos sectores, SAME y CRI, de un hospital público. Con respecto a las acciones desarrolladas por los pasantes, primero se prepararon dos manuales para los sectores, el "Manual CRI de Reglas y Rutinas y Reglas Internas" y el "Manual SAME de Reglas y Rutinas y Reglas Internas". Además, dentro de SAME, los pasantes realizaron actividades en los Sectores de: Archivo, Registro y Hospitalización y Estadísticas, ya dentro del departamento de CRI, las acciones se basaron en la regulación del acceso a los servicios hospitalarios, incluida la gestión de camas y transferencias externas a través de sistemas de información y regulación para camas municipales y estatales. Consideraciones finales: Se concluyó que la pasantía extracurricular proporcionó a los estudiantes una experiencia única, haciéndolos adquirir conocimientos y habilidades que serán de gran valor en sus futuras vidas profesionales.

Palabras-clave: Archivos, Regulación, Enfermería, Organización y administración.

\section{INTRODUÇÃO}

Um hospital eficiente é racionalmente planejado e administrado. A organização juntamente com o planejamento e coordenação é uma das funções básicas da gestão/administração em todas as instituições da sociedade, a atribuição de organizar consiste na atividade complexa de formatação da estrutura organizacional, envolvendo a definição das pessoas, tecnologias, materiais e demais recursos necessários para o alcance dos objetivos de uma determinada instituição, empresa ou organização (LORENZETTI J, et al., 2014).

Ou seja, o administrador deverá ter ciência de tudo que se passa com o paciente, desde o momento em que é admitido, de como estão sendo utilizadas as disponibilidades assistenciais da instituição, da quantidade e qualidade dos serviços e dos custos operacionais (KEDY V, 1984). Nesse contexto, dois setores que auxiliam a gerencia do hospital para o desempenho de um bom trabalho, é o Setor de Arquivo Médico e Estatística (SAME) e o Núcleo Interno de Regulação (NIR), pois, são departamentos que lidam diretamente com a produção de dados e informações técnicas e estatísticas acerca da assistência e com a organização do acesso ao serviço de saúde hospitalar.

Todas as instituições públicas ou privadas produzem e recebem diariamente diversos documentos e devem manter acessíveis as informações, as quais devem ser devidamente armazenadas. O departamento responsável pela ação anteriormente citada é o SAME, que tem como função organizar, armazenar, guardar e gerar estatísticas de prontuários médicos, permitindo sua localização sempre que necessário, seja pela auditoria administrativa ou auditoria de convênios, atender às solicitações de pacientes, secretarias de saúde, vigilância epidemiológica, responder pedidos judiciais, entre outros (SILVA AA, 2012).

Em um hospital organizado, o SAME é a chave do fornecimento exato de uma série de informações relacionados à saúde do paciente. Ele representa, em uma analogia ao organismo humano, o coração do hospital, caso os demais serviços funcionem harmonicamente, ele será o reflexo deste funcionamento (SILVA AA, 2012). Por conseguinte, aliado ao SAME, o NIR é outro departamento de suma importância para a organização e gestão hospitalar.

Para a gestão, o processo regulatório favorece a resolução dos casos de forma eficiente, permitindo um conhecimento mais aprofundado e dinâmico de sua rede de saúde, e favorecendo a identificação de área críticas e das necessidades de saúde de maneira ampliada (RODRIGUES LCR e JULIANI CMCM, 2015). Esse tipo de processo gera ainda melhor controle sobre os gastos em saúde, melhor utilização dos recursos e qualidade da prestação de serviços de saúde (OLIVEIRA RR, 2010). Nesse âmbito, o NIR é responsável pelo processo regulatório hospitalar, tendo a capacidade de gerar, calcular e monitorar indicadores hospitalares de eficiência, de forma a tomar decisões gerenciais (RODRIGUES LCR e JULIANI CMCM, 2015). 
Ademais, como podemos perceber, os dois setores aqui estudados desenvolvem inúmeras atividades de gestão, o que necessita de uma quantidade considerável de profissionais para o desempenho adequado das funções. Com isso, muitos estabelecimentos disponibilizam processos seletivos para estágio extracurricular para acadêmicos de cursos da área da saúde, com o intuito de estimular esses indivíduos a ajudarem na realização das atividades, e em troca receberão inúmeros conhecimentos e habilidades sobre a prática.

A participação em estágios extracurriculares é muito visada por acadêmicos, uma vez que está ligado a necessidade de se adequar à realidade do mercado de trabalho, participando dessas práticas, o estudante estará ganhando experiência, adquirindo habilidades e competências e construindo um bom currículo, sendo estes aspectos essenciais para se inserir na vida profissional (TAQUETE SR, et al., 2007; LAVALL J e BARDEN JE, 2014).

Dessa forma, o estudo possui como principal objetivo relatar a experiência vivenciada por acadêmicos do curso de Enfermagem em um Estágio Extracurricular no SAME e NIR de um hospital do interior do estado do Pará, onde foram realizadas inúmeras atividades de gerenciamento, organização, coordenação e planejamento.

\section{RELATO DE EXPERIÊNCIA}

Trata-se de um estudo descritivo, do tipo relato de experiência, sobre a atuação de discentes do curso de Enfermagem em um Estágio Extracurricular que foi desenvolvido em dois setores, SAME e NIR, de um Hospital Público, localizado no interior do estado do Pará. O estágio está inserido no Programa de Estágios Extracurriculares do referido hospital. Participaram 8 acadêmicos aprovados em processo seletivo. Subdivididos em duplas, com revezamento das atividades entre os setores. A prática ocorreu no período de março a novembro de 2019, com uma carga horária total de 280 horas, sob coordenação de uma enfermeira.

Primeiramente, a atividade inicial desse estágio foi o processo de elaboração de dois manuais para os setores, o "Manual de Normas e Rotinas e Regimento Interno do NIR" e o "Manual de Normas e Rotinas e Regimento Interno do SAME". Sendo que os dois documentos possuíam o intuito de modernizar e uniformizar os trabalhos desenvolvidos pelos funcionários dos departamentos, por meio do reconhecimento e padronização de normas, rotinas e procedimentos do setor, a fim de garantir a excelência na qualidade dos serviços prestados. Posterior a este momento, passaram a desenvolver as atividades que Ihes eram propostas dentro dos dois departamentos.

O SAME do hospital é divido em três setores, Setor de Arquivo, Setor de Registro e Internação e Setor de Estatístico, sendo que cada departamento possui uma localização e equipe profissional própria dentro do hospital. Quando uma dupla de estagiários era direcionada, pela coordenadora do estágio, a um desses departamentos, eles desenvolviam as atividades sob a supervisão dos funcionários daquele setor. Sendo assim, as atividades desenvolvias, foram:

No Setor de Arquivo, os acadêmicos realizavam atividades de guarda, conservação e disponibilização de prontuários para consultas ambulatoriais. Por consequência, mantinham o sistema de arquivamento com sequência numérica, controlavam toda a movimentação de entrada e saída dos prontuários, realizavam a conferência de todos os prontuários retirados do arquivo e zelavam pela conservação física do prontuário.

No Setor de Registro e Internação, os acadêmicos realizavam atividades de recepção e transmissão de informações para os clientes que procuravam o hospital em busca de atendimento. Por conseguinte, desenvolviam acolhimento humanizado, matriculavam no sistema por meio da coleta e registro de dados de identificação fidedignos os pacientes que adentravam na instituição, por fim, repassavam informações ao público desde que não implicasse em respostas técnicas e sigilosas.

No Setor de Estatística, os estagiários realizavam atividades de coleta, apuração, análise e interpretação dos dados obtidos a partir dos prontuários e outras fontes de informação do hospital. Sendo assim, elaboravam estatística de natureza administrativa e nosológica, emitiam informações sobre as admissões, transferências, altas, óbitos e cirurgias ocorridas mensalmente, tabulavam dados estatísticos para a representação gráfica e repassavam esses dados para profissionais que solicitassem. 
Diferentemente do SAME, que é formado por 3 setores, o NIR do hospital em questão é um departamento único, que assim como o setor anterior possui equipe multiprofissional e localização própria dentro da instituição.

A atuação dos acadêmicos no NIR estava relacionada não somente ao fortalecimento do trabalho, mas também à aprendizagem no que tange às atribuições do setor. Vale ressaltar, que todas as atividades desenvolvidas pelos alunos eram supervisionadas por funcionários que compunham o NIR. Mediante a isso, suas ocupações eram definidas baseadas no gerenciamento de leitos, normatizar/monitorar o acesso às dependências da instituição e auxílio no gerenciamento das interfaces externas das Redes de Atenção à Saúde (RAS).

No entanto, a primeira atividade desenvolvida foi a reorganização das cirurgias eletivas, as quais seriam feitas a partir de prioridades de pacientes que estavam na fila de espera e aguardavam desde 2007. Tendo em vista que o hospital é referência para toda a região vizinha do município em que está localizado, houve uma sobrecarga de atendimentos desde o período que estavam necessitando dos procedimentos. Nesse sentido, cada solicitação de operação foi separada de acordo com o município de origem, para que a regulação do local entrasse em contato com os pacientes e repassasem novamente para o NIR do hospital, para que então fosse realizado o agendamento do preparo cirúrgico até o processo propriamente dito.

Posterior a essa atividade de reorganização das cirurgias eletivas, os estagiários passaram a desenvolver das demais funções que lhes eram propostas, o gerenciamento dos leitos ocorria inicialmente em forma de visitas no hospital. O estagiário acompanhado de seu supervisor verificava se os pacientes que foram admitidos admitidos em duas anteriores permaneciam nos leitos ou já haviam recebido alta, de forma que passavam para confirmar nos quartos das clínicas: cirúrgica, médica, psiquiátrica, maternidade, UTI (adulto e infantil) e neonatal. Em seguida, os leitos que eram vagos poderiam ser anotados em um quadro que permanecia no setor para outros profissionais informarem ao Pronto Socorro do hospital e aos municípios que estavam solicitando leito.

A normatização/monitoramento ao acesso as dependências da instituição foram apresentados aos alunos a partir do Sistema Estadual de Regulação (SER) e Sistema de Pactuação entre municípios. O manuseio da plataforma podia ser observado pelos acadêmicos, a qual possibilita a visualização de leitos solicitados e é utilizada pelo setor.

Ademais, os acadêmicos também puderam observar as funções de gerenciamento atribuída aos enfermeiros do setor baseado nas RAS. Na qual, a atuação do profissional está relacionada à avaliação de disponibilidade de leitos, auxiliar a equipe do NIR, interação multidisciplinar assistencial, além de permitir espaços de atuação do enfermeiro em projetos de pesquisa e extensão, objetivando a produção acadêmica e qualificação profissional. Tais atribuições visualizadas no período de estágio puderam dar uma noção básica sobre gerenciamento, tornando uma possível opção para a especialização dos estudantes.

Por fim, nota-se a fundamental importância da experiência proporcionada aos acadêmicos, tendo em vista possuir uma carga horária alta, o que garantiu o desenvolvimento de habilidades, competências e noções acerca de gerenciamento hospitalar, garantindo com que estes estagiários estejam preparados a conduzir e executar diversos procedimentos quando estiverem formados e atuando no mercando de trabalho.

\section{DISCUSSÃO}

A vivência dos acadêmicos frente ao desenvolvimento de atividades de planejamento, coordenação e organização, possibilitou a eles assimilar habilidades e conhecimentos acerca de dois departamentos de hospitais que, geralmente, não recebem tanta atenção por parte da população, dado que quando um indivíduo pensa acerca de um hospital vem em sua mente, automaticamente, a questão assistencial, fazendo-Ihes muitas vezes esquecer os setores de gestão e planejamento, nesse caso SAME e NIR, que são fundamentais para o bom desenvolvimento do todo. Uma gestão mais efetiva sobre os recursos é capaz de ampliar os sistemas de saúde e proporcionar atendimentos de melhor qualidade (BORBA GS, KLIEMANN NETO FJ, 2008). 
No que diz respeito a percepção dos acadêmicos acerca dos dois setores ao final do estágio, podemos dizer que evidenciaram a extrema importância do trabalho do SAME para o hospital, pois sem este serviço devidamente qualificado e estruturado, a instituição poderia sofrer perdas irreparáveis, haja vista que não constará em sua memória informações importantes sobre os pacientes.

O SAME é considerado uma fonte de informação que contribui diretamente com as atividades de qualquer unidade de saúde, dado que parte do princípio de registrar toda e qualquer informação do paciente visando pesquisas futuras (FRANÇA PZP, 2014).

Em referência ao NIR, concluíram que, juntamente com o SAME, é de suma importância para o bom funcionamento do hospital, dado que essa forma de gestão de leitos possibilitará melhor distribuição das internações com otimização da utilização dos leitos e consequente redução do tempo médio de permanência hospitalar e redução da superlotação.

O gerenciamento do acesso à capacidade instalada hospitalar, em um amplo sentido, define o papel geral de um NIR, gerando potencial para o desenvolvimento de ações que resultem em uso eficiente da capacidade instalada hospitalar (SOARES VS, 2017).

Como já foi dito, os dois setores proporcionaram aos estagiários importantes saberes e aptidões acerca de atividades de gerenciamento hospitalar, o que é imprescindível para estes indivíduos que serão futuros enfermeiros, e como sabemos, a gerência é uma das inúmeros funções que este profissional pode desempenhar (COELHO MF, et al., 2010).

Essa vivência se caracterizou como uma oportunidade de preparo para que futuramente possam exercer suas atividades atribuídas de forma correta e eficaz. Por meio de bons conhecimentos acerca de gerenciamento, o enfermeiro constrói um ambiente de trabalho satisfatório, uma vez que será capaz de tomar decisões, resolver problemas, coordenar equipes, planejar, empreender (SOARES MI, et al., 2016).

Ademais, algo importante a ser dito é que os acadêmicos selecionados para participarem do estágio, eram em sua maioria de semestres iniciais do curso de Enfermagem, sendo assim, possuíam pouca ou nenhuma experiência no âmbito do gerenciamento. Tal situação, fez que inicialmente os discentes se deparassem com alguns desafios, mas felizmente, conseguiram superá-los, dado que se adaptaram e utilizaram da criatividade para desenvolverem suas funções.

O estágio é entendido como um importante instrumento para a formação dos profissionais de enfermagem, no qual se desenvolvem habilidades profissionais e se aperfeiçoam técnicas e procedimentos realizados diariamente no exercício da profissão (DIAS EP, et al., 2014).

Ainda, é fundamental que estágios como esse sejam desenvolvidos mais vezes e em outras instituições, pois segundo Restelatto MTR e Dallacosta FM (2018) é necessário incentivar os acadêmicos de enfermagem para o desenvolvimento de competências para a liderança e processos de gerenciamento, pois estas funções são essenciais na integração e inserção do profissional na equipe. Felizmente, a vivência dos estagiários no SAME e no NIR do hospital possibilitou a familiarização deles com essas habilidades.

Por conseguinte, esta vivência não obrigatória para os discentes do curso de Graduação em Enfermagem é de grande valia, dado que thes foi proporcionado experiência para o futuro mercado de trabalho que enfrentarão. Ainda, foi importante para mostrar aos acadêmicos que eles são capazes de desenvolver inúmeras atividades, dado que no começo da experiência estavam receosos, mas conseguiram finalizar com excelência, isso representou uma conquista pessoal para todos.

Nesse contexto, as atividades de estágio não se limitam apenas ao aperfeiçoamento das técnicas e procedimentos, mas tem, como intuito, desenvolver no aluno a capacidade de entendimento pessoal, auxiliando-o a reconhecer e manifestar a sua própria identidade profissional (DIAS EP, et al., 2014).

Segundo Silva ANC, et al. (2019) o estágio extracurricular serve como uma fonte enriquecedora de conhecimento, no futuro, quando os acadêmicos estiverem à procura de seu primeiro emprego, a comprovação de já haverem realizado essa prática pode vir a ser o elemento decisivo para a sua contratação. 
Melo ECGS, et al. (2019) e Silva HRS, et al. (2020), em seus estudos, fazem reflexões acerca da importância que um estágio pode trazer para a instituição que o oferece, bem como para quem os pratica, o discente fica suscetível a desenvolver seu conhecimento teórico e prático, o que fortalecerá no seu aprendizado.

Conclui-se que a participação em estágios extracurriculares proporcionou aos acadêmicos uma experiência ímpar, já que puderam vivenciar a rotina de dois setores de um hospital público, pouco mencionados pelos profissionais e população. Foram 8 meses de atividades, que à priori, provocaram certo receio nos estudantes, tendo em vista que a maioria era de períodos iniciais do curso de Enfermagem.

Porém, se adaptaram rapidamente as rotinas, desenvolveram as atividades de organização, planejamento e coordenação com criatividade o que favoreceu a aplicação prática dos conhecimentos obtidos na graduação que serão de grande valia em suas vidas profissionais futuras.

\section{REFERÊNCIAS}

1. BORBA GS, KLIEMANN NETO FJ. Gestão Hospitalar: identificação das práticas de aprendizagem existentes em hospitais. Saúde e Sociedade, 2008; 17(1): 44-60.

2. COELHO FM, et al. Analysis of the Organizational Aspects of a Clinical Emergency Department: A Study in a General Hospital in Ribeirão Preto, SP, Brazil. Revista Latino-Americana de Enfermagem, 2010; 18(4): 770-777.

3. DIAS EP, et al. Expectativas de alunos de enfermagem frente ao primeiro estágio em instituições de saúde. Revista Psicopedagogia, 2014; 31(94): 44-55.

4. FRANÇA PZP. Reestruturação do Serviço de Arquivo Médico e Estatística do Centro Municipal de Referência em Saúde Leonard Mozart da prefeitura municipal de Cabedelo. Trabalho de Conclusão de Curso (Graduação em Arquivologia) - Centro de Ciências Sociais Aplicadas. Universidade Federal da Paraíba, 2014; 58p.

5. KEDY V. Implantação do Serviço de Arquivo Médico e Estatística em um Hospital Universitário. Dissertação (Mestrado em Saúde Pública) - Faculdade de Saúde Pública. Universidade de São Paulo, 1984; 93p.

6. LAVALL J, BARDEN JE. Estágio não obrigatório: contribuições para a formação acadêmica e profissional do estudante da UNIVATES. Revista Gestão Universitária da América Latina, 2014; 7(3): 47-68.

7. LORENZETTI J, et al. Organização do trabalho de enfermagem hospitalar: arbodagens na literatura. Texto \& Contexto - Enfermagem, 2014; 23(4): 1104-112.

8. MELO ECGS, et al. Contribuições de um estágio extracurricular para o aprimoramento de habilidades acadêmicas de enfermagem na realização de exames ginecológicos. Revista Estácio Saúde, 2019; 8(2): 12-15.

9. OLIVEIRA RR. Os conceitos de regulação em saúde no Brasil. Dissertação (Mestrado em Ciências) - Faculdade de Medicina. Universidade de São Paulo, 2010; 117p.

10. RESTELATTO MTR, DALLACOSTA FM. Vivências do acadêmico de enfermagem durante o estágio com supervisão indireta. Enfermagem em Foco, 2018; 9(4): 34-38.

11. RODRIGUES LCR, JULIANI CMCM. Resultado da implantação de um Núcleo Interno de Regulação de Leitos nos indicadores administrativos-assistenciais em um hospital de Ensino. Einstein (São Paulo), 2015; 13(1): 96-102.

12. SILVA AA. Estudo do Serviço de Arquivo Médico e Estatística do Hospital São Donato/SC: Resultado da Pesquisa. Monografia (Especialização em Gestão de Arquivos) - Centro de Ciências Sociais e Humanas. Universidade Federal de Santa Maria, 2012; 66p.

13. SILVA ANC, et al. Estágio Extracurricular de Enfermagem: Estratégia para a formação profissional. Enfermagem em Foco, 2019; 10(4): 129-135.

14. SILVA HRS, et al. Práticas de educação em saúde desenvolvidas pelo núcleo de ensino e pesquisa (NEP) de um hospital do interior do Pará. Revista Eletrônica Acervo Saúde, 2020; 12(8): e3640.

15. SOARES MI, et al. Saberes gerenciais do enfermeiro no contexto hospitalar. Revista Brasileira de Enfermagem, 2016; 69(4): 631-637.

16. SOARES VS. Análise dos Núcleos Internos de Regulação hospitalares de uma capital. Einstein (São Paulo), 2017; 15(3): 339-343.

17. TAQUETE SR, et al. Currículo paralelo: uma realidade na formação dos estudantes de medicina da UERJ. Revista Brasileira de Educação Médica, 2007; 31(3): 245-253. 